\title{
Confinement Effect in a Thick Layer of LC Lanthanide Complex ${ }^{+}$
}

\author{
Irina Lezova, Galina Polushina and Sergey Polushin* \\ Department of Physics, St. Petersburg State University, Universitetskaya nab. 7/9, St. Petersburg 199164, \\ Russia; i.lezova@spbu.ru (I.L.); gepol@mail.ru (G.P.) \\ * Correspondence: s.polushin@spbu.ru \\ + Presented at the 1st International Electronic Conference on Crystals, 21-31 May 2018. Available online: \\ https://sciforum.net/conference/IECC_2018. \\ Published: 21 May 2018
}

\begin{abstract}
The structure of metallomesogen complexes differs essentially from that of calamitic liquid crystals, which emerges in their physical properties. We studied anomalous features of the transition from the nematic to isotropic phase in the ytterbium-based liquid crystal complex. For this, two approaches were used. In the first approach, we showed that the experimental values of the dielectric and optical anisotropies measured near the phase transition turned out to be substantially smaller than their calculated values. This evidently occurs because the phase transition temperature in the deep region of the experimental cell differs from that near the surface of the cell. Secondly, the temperature of $\mathrm{N}-\mathrm{I}$ transitions in metallomesogenic layers within glass sandwich cells is obtained directly by means of the polarization microscope method. It was found that the phase transition temperature drops in more than ten degrees when reducing the metallomesogenic layer thickness from 200 to 5 microns. The calamite liquid crystals show the confinement effect only within the interstice of the specific size smaller than one micron. An anomalous confinement effect can be caused by a strong interaction of the molecular complexes with each other.
\end{abstract}

Keywords: phase transition; metallomesogen; confinement

\section{Introduction}

The recent years have witnessed considerable progress in the synthesis and studies of liquidcrystalline lanthanide complexes, owing to the progress in the physics of liquid crystals and in coordination and organic chemistry [1,2]. The molecular structure of complexes is considerably more sophisticated than that of classical liquid crystals. This feature, together with the presence of metal atoms in the complexes, account for their peculiar physical properties; in particular, most metallomesogens are smectics. A typical feature of metallomesogenic complexes is the combination of the optical and electrical anisotropies inherent in typical liquid crystals with the magnetic and electronic properties inherent in transition metal complexes. They exhibit efficient luminescence, and the magnetic susceptibility anisotropy of metallomesogens is orders of magnitude higher than that of organic liquid crystals [3,4]. The successful synthesis of nematic metallomesogens [5] opened up the possibility to obtain and study macroscopically ordered liquid-crystalline phases [6]. Systematic studies of metallomesogens revealed one more feature, which has not been deliberately investigated so far and is analyzed in this work. In particular, the first-order phase transition from the liquidcrystalline to isotropic phases can take place within a temperature range of one to several degrees. This should give rise to the coexistence of the anisotropic and isotropic phases and to effective anisotropy of physical properties being lower in this range than the anisotropy of the proper liquidcrystalline phase. In this work, we compared the electro-optical properties of the metallomesogen in the isotropic (I) phase with the optical and dielectric anisotropy of the metallomesogen determined 
in the nematic $(\mathrm{N})$ phase. The anisotropy measured experimentally near the transition proved to be many times lower than the calculated value.

The cause for phase coexistence was determined by monitoring the phase transition by polarization microscopy. This is the effect of boundaries of the cell containing the sample at the N-I phase transition temperature, i.e., the confinement effect. In the case of classical liquid crystals placed into a thin cell or porous matrix, this effect is manifested if the cell (pore) characteristic dimension is less than a micrometer [7]. The confinement effect found in metallomesogens for layer thicknesses of an order of magnitude greater was a new and unexpected finding.

\section{Methods}

The liquid-crystalline complex studied in this work was tris[1-(4-(4-propylcyclohexyl) phenyl) octane-1,3-diono]-[5,5'-di(heptadecyl)-2,2'-bipyridineytterbium, designated as $\mathrm{Yb}\left(\mathrm{DDk}_{3-5}\right)_{3} \mathrm{Bpy}$ 17-17. It has both smectic $A$ and nematic phases [5].

The electro-optical properties of an isotropic melt above the nematic-isotropic melt phase transition temperature were studied by considering the Kerr effect, which serves as a tool for examining phase transitions in mesogens $[9,10]$. The electric field-induced birefringence $\Delta \mathrm{nE}$ is related to the Kerr constant $\mathrm{K}$ and the electric field strength $\mathrm{E}$ by the Kerr equation: $\Delta \mathrm{nE}_{\mathrm{E}}=\mathrm{KE}^{2}$. The electric birefringence in the isotropic melt was measured in rectangular pulse fields with strengths of up to 5 $\times 10^{3} \mathrm{~V} / \mathrm{cm}$. In order to eliminate the electrical conductivity and parasitic heating effects, short (0.5 ms) electric pulses with a repetition rate of less than $0.1 \mathrm{~Hz}$ were used. The Kerr cell with the sample was maintained at a constant temperature to within $0.1^{\circ} \mathrm{C}$.

For determining the conditions of existence of the two-phase liquid-crystal-isotropic melt system in the $\mathrm{Yb}\left(\mathrm{DDk}_{3-5}\right)_{3} \mathrm{Bpy}$ 17-17 $_{17}$ mesogen, we examined the $\mathrm{N}-\mathrm{I}$ transition in the planar and wedgelike layers of various thickness, $\mathrm{d}$, by polarization microscopy. The samples were sandwiched between clean glasses whose surfaces were not treated in any way. The sample heating and cooling rates did not exceed $0.5 \mathrm{~K} / \mathrm{min}$.

\section{Results and Discussion}

The plot for reciprocal of the Kerr constant 1/K versus temperature T is shown in Figure 1.

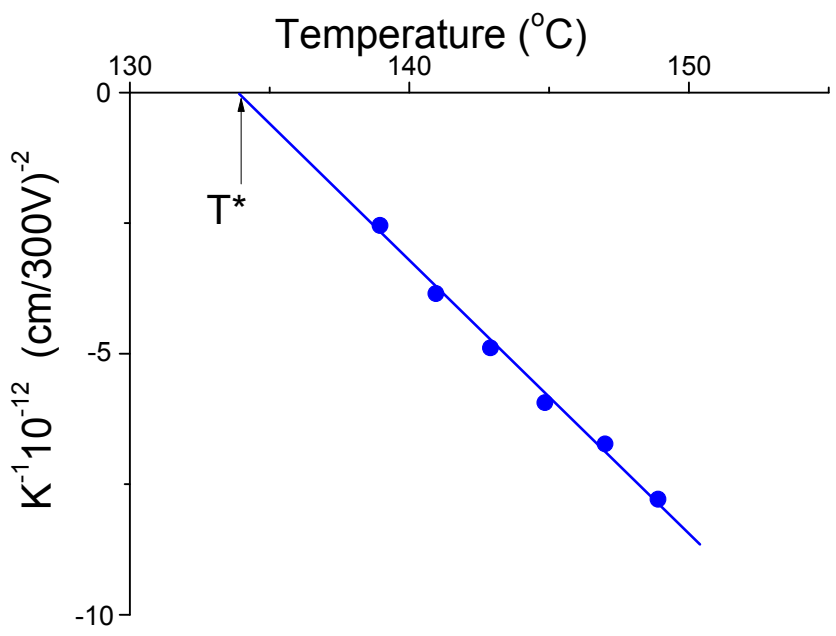

Figure 1. Temperature dependence of the reciprocal of the Kerr constant $1 / \mathrm{K}$ in an isotropic melt of the mesogenic complex $\mathrm{Yb}\left(\mathrm{DDk}_{3-5}\right)_{3} \mathrm{Bpy}$ 17-17. The limiting temperature of existence of the isotropic phase, $\mathrm{T}^{*}$, is designated.

The EBR sign is negative, indicating that the permanent dipole moment of the complex forms an angle of more than $55^{\circ}$ with its longitudinal axis. As the temperature is lowered, the absolute magnitude of the Kerr constant $\mathrm{K}$ increases and the reciprocal of the Kerr constant varies linearly; i.e., the dependence $K \sim 1 /\left(T-T^{*}\right)$ holds. The electro-optical constant $K\left(T-T^{*}\right)$ is $-2.0 \times 10^{-8}(\mathrm{~cm} / 300 \mathrm{~V})^{-2}$ 
K. By extrapolation of the temperature dependence of $1 / \mathrm{K}$, the limiting temperature of existence of the isotropic phase was found to be $\mathrm{T}^{*}=133.9^{\circ} \mathrm{C}$.

A phenomenological approach to the description of the pretransition behavior of a liquid crystal was developed by De Gennes on the basis of the Landau phase transition theory [8]. In the I-N phase transition region, the free-energy density $\mathrm{F}$ in the presence of an electric field of strength $E$ is expanded in the powers of the orientational order $S$, and consideration can be restricted to the quadratic term: $F(T)=F_{0}(T)+\frac{1}{2} a\left(T-T^{*}\right) S^{2}-\frac{1}{12 \pi} \Delta \varepsilon E^{2}$. Here, $F_{0}(T)$ is the energy of the isotropic phase and $\Delta \varepsilon$ is the dielectric anisotropy. The coefficient $a$ in the expansion is related to the heat of melting $L$ in the following way: $L=a T_{c} S_{c}^{2} / 2$. The order parameter $S_{c}$ corresponds to the order at the N-I phase transition temperature $T_{c}$. The Landau-De Gennes theory makes it possible to express the dielectric $\Delta \varepsilon_{c}$ and optical $\Delta n_{c}$ anisotropies of a liquid crystal at temperature $T_{c}$ in terms of the electro-optical constant of the isotropic phase $K\left(T-T^{*}\right)$ in the following way:

$$
\Delta n_{c} \Delta \varepsilon_{c}=24 \pi L K\left(T-T^{*}\right) / T_{c}
$$

Equation (1) adequately describes the behavior of classical liquid crystals [11]. Using the measured electro-optical constant $K\left(T-T^{*}\right)$ and the values $L=8.0 \times 10^{6} \mathrm{erg} / \mathrm{cm}^{3}$ and $T_{c}=127^{\circ} \mathrm{C}$, we calculated the anisotropy from equation (1) to be $\Delta n_{c} \Delta \varepsilon_{c}=-0.03$. An absolutely different result is obtained upon multiplication of the experimentally determined optical [12] and dielectric [6] anisotropy values near the transition: $\Delta n_{c} \Delta \varepsilon_{c}=-0.00024$. Evidently, the presence of the isotropic liquid phase in the anisotropic liquid near the phase transition decreases the value of $\Delta n_{c} \Delta \varepsilon_{c}$ by two orders of magnitude. This can be ascertained by using data on the mesophase anisotropy at low temperature, where an isotropic phase impurity is absent. Using the low-temperature values $\Delta n=0.08$ and $\Delta \varepsilon=$ -1.9 , we converted them to the temperature $T_{c}$, bearing in mind that the temperature dependence of $\Delta n$ and $\Delta \varepsilon$ is a function of the order parameter: $\Delta \varepsilon \sim \mathrm{S}$ and $\Delta n \sim \mathrm{S}$. The usual values of the order parameter $S$ are 0.7 in the low-temperature region and 0.4 in the close vicinity of $T_{c}$; from this, we find that the anisotropy decreases 1.75-fold near the transition, and then $\Delta n_{c} \Delta \varepsilon_{c}=-0.05$. This result is already comparable with the value calculated from Equation (1). Thus, combined analysis of optical, dielectric, and electro-optical data indicates that the isotropic and anisotropic phases coexist in the vicinity of phase transition.

The specific behavior of metallomesogens in the N-I phase transition is manifested most clearly in the case of a cell of variable thickness; this is demonstrated by the micrograph in Figure 2.

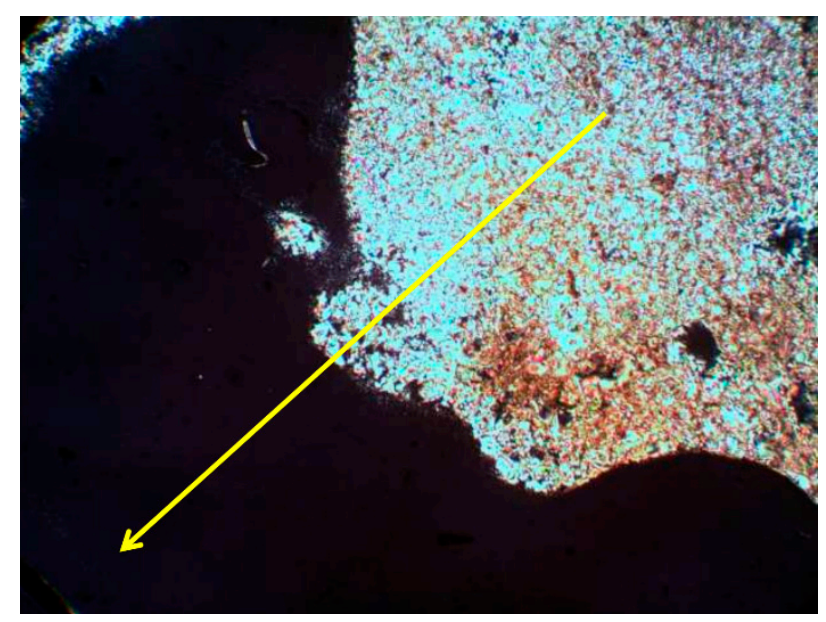

Figure 2. Photomicrograph $(80 \times)$ of a metallomesogen layer at $126.0^{\circ} \mathrm{C}$. The polarizers are crossed and the isotropic phase looks black. The arrow shows the direction in which the layer thickness linearly decreases from $200 \mu \mathrm{m}$ to $5 \mu \mathrm{m}$. The N-I phase interface moves along the same direction with temperature change in the interval of $118-130^{\circ} \mathrm{C}$. 
As the isotropic melt is cooled, the formation of the liquid-crystalline phase starts at temperature $\mathrm{T}_{1}$ from the region in which the layer thickness $\mathrm{d}=200 \mu \mathrm{m}$. With decreasing temperature, the liquid crystal-isotropic phase boundary moves along the arrow towards part of the sample where $d=5 \mu \mathrm{m}$. Thus, the phase transition occurs in a $\sim 10{ }^{\circ} \mathrm{C}$ temperature interval between $T_{1}$ and $T_{2}$, where $T_{1}$ is the higher temperature at which the mesophase is formed deep in the layer and $T_{2}$ is the minimal temperature at which the nematic phase is formed in the narrow part of the layer. The phase transition under the same conditions for a conventional calamitic occurs in an interval of less than 0.1 degrees; see Figure 3.

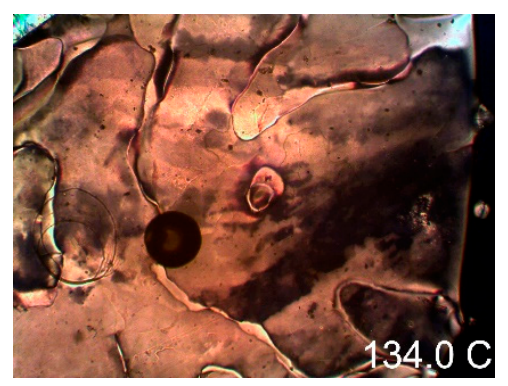

(a)

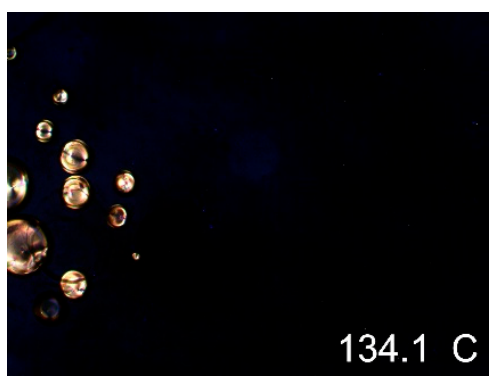

(b)

Figure 3. The phase transition in classical PAA ( $p, p^{\prime}$-di- $n$-methyloxyazoxybenzen) in the same cell occurs in the range of about $0.1^{\circ} \mathrm{C}$ or less. Here, photomicrograph (a) was made at $134.0 \mathrm{C}$ and (b) at $134.1 \mathrm{C}$.

In 8- and 50- $\mu \mathrm{m}$ thick planar layers, the temperature interval $\mathrm{T}_{1}-\mathrm{T}_{2}$ narrows down to reach a minimal value of about $1{ }^{\circ} \mathrm{C}$ in the thinnest layer, as one can see in the plot in Figure 4 . The $\mathrm{T}_{2}$ value is almost invariable as it characterizes the transition near the boundaries, whereas $\mathrm{T}_{1}$ substantially depends on thickness. As follows from the plot, the curve should arrive at saturation near $d=1000$ $\mu \mathrm{m}$ upon reaching the limiting temperature $\mathrm{T}^{*}$ of the isotropic phase existence; for a nematic, $\mathrm{T}^{*}$ differs from the temperature of the $\mathrm{N}-\mathrm{I}$ transition in the bulk by less than one degree: $\mathrm{T}^{*} \approx \mathrm{T}_{\mathrm{c}}$. In other words, the difference $\mathrm{T}^{*}-\mathrm{T}_{2}=14^{\circ} \mathrm{C}$ corresponds to the greatest transition temperature shift for the metallomesogen $\mathrm{Yb}\left(\mathrm{DDk}_{3-5}\right)_{3} \mathrm{Bpy} 17-17$ under the boundary conditions specified in the experiment.

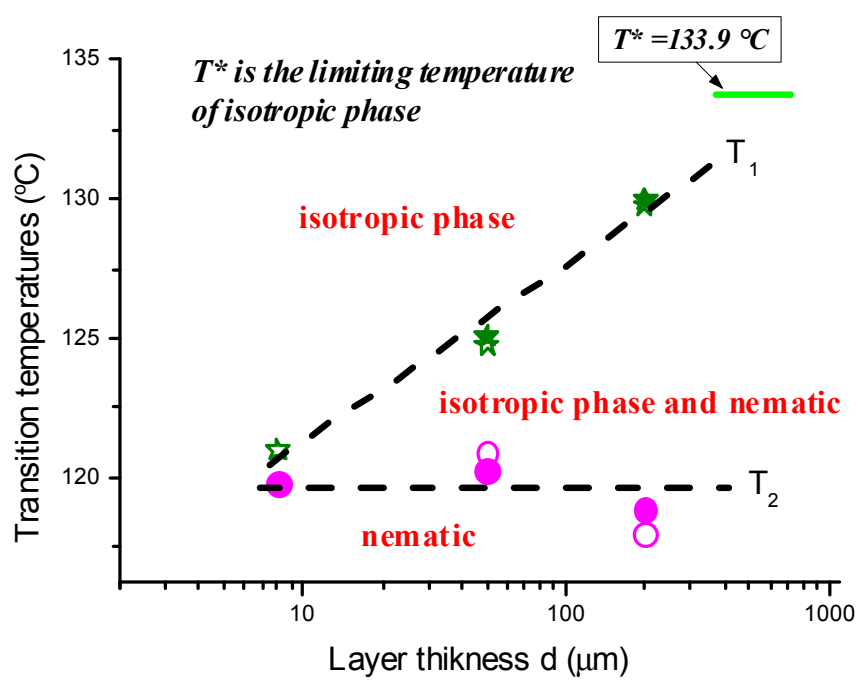

Figure 4. Phase diagram of a metallomesogen obtained using three samples with different layer thicknesses: 8,50 , and $200 \mu \mathrm{m}$. The nematic and isotropic phase regions and the coexistence region are designated. The temperature $\mathrm{T}^{*}$ was determined by using the Kerr effect. During cooling (dark dots), the nematic-isotropic phase transition starts at the temperature $\mathrm{T}_{1}$ and ends at $\mathrm{T}_{2}$. During heating (light dots), melting of the nematic starts at the temperature $\mathrm{T}_{2}$ and ends at $\mathrm{T}_{1}$. The green dots correspond to the transition temperature at the center of the cell, while the pink dots correspond to the transition temperature near the glass surface. 
The shift of the N-I transition temperature and, in some cases, also the transformation of the first-order phase transition into a continuous transition are caused by the influence of the extensive boundary on the order parameter in the melt [13]. It was found in experiments using thin layers of liquid-crystalline alkyl cyanobiphenyl with one free surface that the shift is determined by a combination of three factors: surface tension, boundary anchoring, and capillary condensation [7]. The shift increases as the characteristic size $d$ decreases and the shift can be both positive and negative. A computer modeling of the $\mathrm{N}-\mathrm{I}$ transition under confinement [14] demonstrated that there is no single universal scenario that would describe the transition properties as a function of $d$; the transition behavior being dependent only on intermolecular interaction details. This conclusion is consistent with our assumptions that the probable causes of the above-described phase behavior are related to the sophisticated molecular architecture of metallomesogenic complexes. The result of this study is practically significant, because it implies that the confinement effects can be both manifested under certain conditions and used in the existing electro-optical sensors, modulators, and other devices.

Author Contributions: S.P. conceived and designed the experiments; G.P. and I.L. performed the experiments and analyzed the data; S.P. wrote the paper.

Acknowledgments: The authors are grateful to V. B. Rogozhin and E. I. Rjumtsev for assistance in the work, and are grateful to Yu. G. Galyametdinov for providing the sample.

Conflicts of Interest: The authors declare no conflict of interest.

\section{Abbreviations}

The following abbreviations are used in this manuscript:

EBR electric birefringence

\section{References}

1. Serrano, J.L. Metallomesogenes, Synthesis, Properties and Applications; VCH: New York, NY, USA, 1996; doi:10.1002/9783527615094.

2. Binnemans, K.; Gorller-Walrand, C. Lanthanide-containing liquid crystals and surfactants. Chem. Rev. 2002, 102, 2303-2345.

3. Knyazev, A.A.; Molostova, E.Y.; Krupin, A.S.; Heinrich, B.; Donnio, B.; Haase, W.; Galyametdinov, Y.G. Mesomorphic behaviour and luminescent properties of mesogenic-Diketonate lanthanide adducts with 5,5'-di(heptadecyl)-2,2'-bipyridine. Liq. Cryst. 2013, 40, 857-863, doi:10.1080/02678292.2013.795626.

4. Galyametdinov, Y.G.; Haase, W.; Malykhina, L.; Prosvirin, A.; Bikchantaev, I.; Rakmatullin, A.; Binnemans, K. Synthesis, mesomorphism, and unusual magnetic behavior of lanthanide complexes with perfluorinated counterions. J. Chem. Eur. 2001, 7, 99-105.

5. Dzhabarov, V.I.; Knyazev, A.A.; Strelkov, M.V.; Molostova, E.Y.; Schustov, V.A.; Haase, W.; Galyametdinov, Y.G. Tris( $\beta$-diketonates) lanthanum nematic adducts. Liq. Cryst. 2010, 37, 285-291, doi:10.1080/02678290903506040.

6. Dobrun, L.A.; Sakhatskii, A.S.; Kovshik, A.P.; Ryumtsev, E.I.; Knyazev, A.A.; Galyametdinov, Y.G. Dielectric properties of an ytterbium_based nematic liquid_crystal complex. JETP Lett. 2014, 99, 133-135, doi:10.1134/S0021364014030072

7. Wittebrood, M.M.; Luijendijk, D.H.; Stallinga, S.; Rasing, T.; Musevic, I. Thickness-dependent phase transition in thin nematic films. Phys. Rev. E 1996, 54, 5232-5234.

8. De Gennes, P.G.; Prost, J. The Physics of Liquid Crystals; Oxford University Press: New York, NY, USA, 1993.

9. Polushin, S.; Rogozin, V.; Beloborodov, I.; Rjumtsev, E.; Kozlovsky, M. Existence of two different isotropic phases as a reason for bistable phase behavior of an LC side-chain polymethacrylate. Macromol. Rapid Commun. 2008, 29, 224-228, doi:10.1002/marc.200700638.

10. Rogozhin, V.B.; Lezov, A.V.; Polushin, S.G.; Ryumtsev, E.I. Effect of the microphase separation on the electro-optical properties of isotropic melts of thermotropic liquid crystals. Zhurnal Fizicheskoi Khimii 2011, 85, 2005-2010, doi:10.1134/S0036024411110276.

11. Coles, H.J. Laser and electric field induced birefringence studies on the cyanobiphenyl homologues. Mol. Cryst. Liq. Cryst. 1978, 49, 67-74. 
12. Kovshik, A.P.; Krainyukov, E.S.; Kovshik, S.A.; Knyazev, A.A.; Galyametdinov, Y.G.; Ryumtsev, E.I. Optical anisotropy of liquid_crystal lanthanide complexes. Opt. Spektrosk. 2014, 116, 61-67, doi:10.1134/S0030400X1401010X.

13. Sheng, P. Phase transition in surface-aligned nematic films. Phys. Rev. Lett. 1976, 37, 1059-1062.

14. Fish, J.M.; Vink, R.L.R. Isotropic-to-nematic transition in confined liquid crystals: An essentially nonuniversal phenomenon. Phys. Rev. E 2010, 81, 021705, doi:10.1103/PhysRevE.81.021705.

(C) 2018 by the authors. Licensee MDPI, Basel, Switzerland. This article is an open access article distributed under the terms and conditions of the Creative Commons Attribution (CC BY) license (http://creativecommons.org/licenses/by/4.0/). 